\title{
Caracterização da acessibilidade aos empregos percebida pelos usuários do sistema metroferroviário da região metropolitana de Fortaleza
}

\author{
Hélio Henrique Holanda de Souza ${ }^{1}$, Francisco Moraes de Oliveira Neto ${ }^{2}$, \\ Carlos Felipe Grangeiro Loureiro ${ }^{3}$ \\ ${ }^{1}$ Agência Reguladora do Estado do Ceará, Instituto Federal de Educação, Ciência e Tecnologia do Ceará \\ ${ }^{2}$ Programa de Pós-graduação em Engenharia de Transportes, Universidade Federal do Ceará \\ ${ }^{3}$ Programa de Pós-graduação em Engenharia de Transportes, Universidade Federal do Ceará
}

\section{Recebido:}

12 de setembro de 2018

Aceito para publicação:

4 de fevereiro de 2020

Publicado:

30 de abril de 2020

Editor de área:

Helena Beatriz Cybis

\section{Palavras-chaves:}

Acessibilidade,

Escolha Discreta,

Logsum,

Indicador,

Caracterização.

\section{Keywords:}

Accessibility,

Choice,

Logsum,

Indicator,

Description.

DOI:10.14295/transportes.v28i1.1836

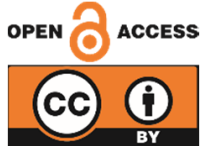

\begin{abstract}
RESUMO
A mudança de paradigma para um planejamento dos transportes com foco na acessibilidade, associada às críticas sobre as técnicas tradicionais de avaliação ex-ante, abriram espaço para novas abordagens de quantificação dos impactos diretos e indiretos advindos das alternativas de intervenção, a partir de medidas de acessibilidade baseadas no conceito da utilidade percebida pelos usuários. Neste contexto, este trabalho objetiva caracterizar os benefícios da implantação do sistema metroviário na melhoria das condições de acesso aos empregos da população de baixa renda da Região Metropolitana de Fortaleza (RMF), por meio da proposição de um método baseado na modelagem do valor do tempo e em indicador do tipo logsum que incorpora a percepção dos usuários com relação à sua problemática específica. Como principal contribuição metodológica, destaca-se o uso da modelagem comportamental no esforço analítico de melhor compreensão da problemática da acessibilidade. Ademais, a aplicação do método permitiu caracterizar espacialmente os ganhos de acessibilidade com as linhas Sul e Oeste do Metrofor, destacando os principais fatores, segundo a percepção dos indivíduos que utilizam o sistema, que podem explicar a variação dos benefícios dessa intervenção na RMF.
\end{abstract}

\section{ABSTRACT}

The paradigm shift towards transport planning focused on accessibility, together with the criticism on traditional techniques for ex-ante assessment, opened up new approaches for quantifying direct and indirect impacts of alternative solutions, by the use of accessibility measures based on the concept of users' perceived utility. In such a context, this work aims to characterize the benefits of implementing the metro system in enhancing the access conditions to jobs for the low-income population in Fortaleza's Metropolitan Region (RMF), through the proposition of a method based on modeling travel time and on a logsum-type indicator which incorporates users' perception of its specific problem setting. Its main methodological contribution is the use of behavioral modeling in the analytical effort of better understanding accessibility problems. Moreover, the method application allowed to spatially characterize the accessibility gains of Metrofor's South and West lines, highlighting the main factors, according to the system's users perception, which could explain the variation of its benefits over the RMF.

\section{INTRODUCÃO}

A demanda por viagens deriva da necessidade de acessar as funções e serviços urbanos em lugares diferentes, sendo determinada pela distribuição espacial dos domicílios e das atividades 
no território urbano (Cascetta, 2009). Essa decisão de se deslocar está associada a outras decisões, de curto e longo prazo, à disponibilidade da oferta de transportes e às características dos subsistemas de atividades e de uso do solo (Lopes et al., 2018). Trata-se de um fenômeno complexo cuja compreensão da problemática, hierarquização de objetivos, proposição e avaliação de soluções compreendem o escopo do processo de planejamento urbano integrado (Soares et al., 2018), reinterpretado nas últimas duas décadas como o novo paradigma de planejamento da acessibilidade e mobilidade urbanas (Handy, 2005; Banister, 2008; Garcia et al., 2018), requerendo uma melhor reflexão sobre as medidas que representam o afastamento espacial entre as atividades e as pessoas que querem acessá-las (van Wee, 2016).

Diversas são as possibilidades de intervenção nos subsistemas de uso do solo e transportes de modo a promover melhor acessibilidade ofertada ou percebida pela população (Geurs et al., 2010). Entre elas se destaca a implementação de sistemas de metrô que durante anos foi vista como única intervenção de transporte de massa capaz de contribuir efetivamente para melhorar as condições de acessibilidade e mobilidade urbanas. Porém, o alto custo envolvido e o surgimento de outras alternativas de mais rápida e eficiente implantação, como o BRT, colocaram em xeque a antiga unanimidade do modo metroviário, suscitando a discussão de como avaliar mais adequadamente essas intervenções.

Tradicionalmente, tem-se utilizado o método de benefício-custo, com o valor monetizado do tempo assumindo relevante papel na comparação do retorno dado aos distintos segmentos da sociedade (Small e Rosen, 1981). Entretanto, a mudança de paradigma para um planejamento integrado com foco na acessibilidade, associada às críticas sobre as técnicas tradicionais de avaliação, abriram espaço para novas abordagens de quantificação dos impactos diretos e indiretos advindos de cada alternativa, a partir de medidas de acessibilidade baseadas no conceito da utilidade percebida pelos usuários, como o logsum (Handy e Niemeier, 1997; Jong et al., 2007; Geurs et al., 2010).

Neste contexto, o presente trabalho tem como objetivo geral caracterizar a contribuição da implantação do sistema metroferroviário de Fortaleza (Metrofor), considerando o seu estágio de operação em agosto de 2018, na melhoria das condições de acessibilidade aos empregos da população de baixa renda da Região Metropolitana de Fortaleza (RMF), por meio da proposição de um método baseado na modelagem do valor do tempo e em um indicador do tipo logsum (Souza e Loureiro, 2018), de modo a incorporar na avaliação a percepção dos usuários com relação à sua problemática específica.

\section{LACUNAS DE PESQUISA SOBRE MEDIDAS DE ACESSIBILIDADE URBANA}

Como medir acessibilidade a atividades, entendida como potencial de oportunidades de interação entre o homem e o meio (Hansen, 1959), vem sendo tema de pesquisa há bastante tempo. Entretanto, ainda existem diversos desafios conceituais e metodológicos na mensuração da acessibilidade, como discutem Geurs et al. (2014), van Wee (2016) e Miller (2018). De um lado estão questões relacionadas a atributos objetivos sobre as opções ofertadas pelos sistemas de transportes e de uso do solo. Van Wee (2016) e Miller (2018) alertam que medidas objetivas não consideram de forma apropriada a variedade de opções de transportes conforme o propósito de viagem e nem a diversidade de atividades que possam existir no meio urbano. Geurs et al. (2014) destacam também questões intrínsecas à relação dinâmica entre uso do solo e acessibilidade, assim como a relação da acessibilidade com a topologia das redes de transportes. Por outro lado, há uma discussão sobre a consideração da percepção que os indivíduos têm sobre 
os fatores que influenciam a acessibilidade e as diferenças de percepção dentro e entre grupos sociais. A importância de reconhecer a acessibilidade percebida pelos indivíduos como determinante real do comportamento tem sido enfatizada por muitos pesquisadores (Koenig, 1980; Cascetta et al. 2013; van Wee, 2016), argumentando que a acessibilidade somente se concretiza quando se consideram as preferências e restrições dos indivíduos que surgem devido à utilização do sistema.

Miller (2018) discute ainda problemas conceituais e práticos encontrados na avaliação de políticas públicas e no planejamento de transportes devido à falta de fundamentação teórica para se definir medidas de acessibilidade. Uma questão importante é de como definir o que pode ser considerado como nível aceitável de acessibilidade para um dado grupo social. Outra questão é que a acessibilidade (como um potencial de interação) é uma consequência do estado atual dos sistemas de transportes e de uso do solo, podendo ser medida apenas de forma relativa dentro de um mesmo grupo social e num dado período específico. Portanto, como medir a evolução da acessibilidade ao longo do tempo e as diferenças de acessibilidade entre grupos sociais são questões ainda não bem resolvidas na literatura. Miller (2018) ainda destaca dificuldades em avaliar hipóteses causais e modelos dos efeitos da acessibilidade sobre o uso do solo (escolha locacionais de residências, oportunidades de emprego e valor de terra) já que, em geral, medidas de acessibilidade são obtidas a partir de dados transversais de um dia típico (dados estáticos sobre o estado atual do sistema) com a premissa de que os sistemas estão em equilíbrio. Assim, um suposto efeito dinâmico do uso do solo sobre o sistema de transporte não poderia ser analisado.

\section{INDICADORES PARA CARACTERIZAÇÃO DA ACESSIBILIDADE}

Visando contribuir com algumas das lacunas de pesquisa, Souza e Loureiro (2018) propuseram medidas para caracterizar problemas de acessibilidade no meio urbano com base na teoria da utilidade aleatória. Os autores definiram um conjunto de indicadores de acessibilidade que incorporam o valor subjetivo de se ter múltiplas opções de transportes (modos ou rotas) e múltiplas opções de atividades no meio urbano (variedades de usos do solo), o que não pode ser capturado por medidas objetivas de acessibilidade como o tempo de viagem e o valor numérico da quantidade de oportunidades. A formulação dos indicadores além de estar fundamentada na modelagem das preferências dos indivíduos sobre as opções de transportes também tem como base uma tipologia de problemas baseada nos princípios da equidade e sustentabilidade proposta por Garcia et al. (2018). Conforme discutido por Souza e Loureiro (2018), Garcia et al. (2018) sugerem quatro tipos de problemas de acessibilidade: Problema tipo I - desigualdade espacial da acessibilidade para um dado grupo social; Problema tipo II - desigualdade nos níveis de acessibilidade entre diferentes grupos sociais; Problema tipo III - diferenças de acessibilidade que podem ocorrer devido às diferentes ofertas e caraterísticas dos modos de transportes; e Problema tipo IV - que se refere a evolução da problemática da acessibilidade ao longo das gerações de usuários.

O conjunto de indicadores proposto por Souza e Loureiro (2018) para caracterizar a acessibilidade no nível estratégico do planejamento de transportes e uso do solo tem como base a formulação da Equação 1, a seguir:

$$
A_{o}=\frac{\sum_{d=1}^{Z}\left(\frac{1}{\alpha_{n}} \ln \sum_{i} e^{V_{i n}}\right) \times E M P_{d} \times P O P_{o}}{\sum_{d=1}^{Z} E M P_{d} \times \sum_{o=1}^{Z} P O P_{o}}
$$


Em que $A_{o}$ representa a acessibilidade da zona de origem $o ; E M P_{d}$ o número de empregos na zona de destino $d$, ou outro atributo que represente a atratividade de interesse; $P O P_{o}$ a população economicamente ativa da zona de origem $o ; z$ o total de zonas da região em estudo; $\ln \sum_{i} e^{V_{\text {in }}}$ é um indicador de acessibilidade de um indivíduo $n$ conhecido como logsum, cuja formulação é obtida a partir da modelagem das preferências dos usuários sobre as opções de transportes a partir de modelos do tipo logit, que é o modelo mais comum de escolha discreta (veja Louviere et al., 2010; e Train, 2009); $V_{\text {in }}$ é a parcela sistemática da utilidade da alternativa $i$, que pode ser definida como uma função de vários atributos do deslocamento e dos usuários; e $\alpha_{n}$ é a utilidade marginal da renda de um indivíduo $n$, usada para transformar o logsum em uma medida monetária.

Essa acessibilidade "total" representa uma medida de impedância de uma determinada zona de origem $o$ a todas as outras zonas, ponderada por uma medida de atratividade das zonas de destino e por uma medida que representa a parcela de indivíduos de determinado grupo social na zona de origem competindo por atividades na zona de destino. Embora seja possível incorporar a percepção dos indivíduos sobre as opções de atividades no destino, a formulação proposta por Souza e Loureiro (2018) assume que o efeito das opções de atividades é proporcional a distribuição espacial dos empregos no meio urbano para um dado grupo de indivíduos.

Como visto na Equação 1, Souza e Loureiro (2018) definiram indicadores tendo como base a formulação do logsum, uma medida comumente usada na etapa de avaliação de alternativas de intervenção do sistema de transportes (Geurs e van Wee, 2004; Jong et al., 2005; Jong et al., 2007; Geurs et al., 2010; van Wee, 2016). 0 logsum é uma medida agregada subjetiva sobre a satisfação de um grupo social sobre um dado sistema em que várias escolhas são possíveis. Uma medida similar é o valor do tempo como sendo a disposição a pagar por redução dos tempos de deslocamento no sistema de transportes. O logsum é também interpretado como uma medida do excedente do consumidor ou da variação do bem-estar proporcionado por uma intervenção ou política pública de transporte ou uso do solo. Um indicador baseado no logsum, portanto, contabiliza tanto as mudanças nos custos generalizados de transporte e na utilidade dos destinos, como é capaz de mensurar os benefícios na acessibilidade de mudanças na distribuição das atividades devido a políticas de transportes ou uso do solo (Geurs et al., 2010).

Como medida de acessibilidade, o logsum mede a satisfação percebida pelos usuários dada uma determinada escolha frente a um conjunto de alternativas (Niemeier, 1997; Bhat et al., 2000; Börjesson et al., 2014; Geurs et al., 2010; van Wee, 2016), apresentando portanto potencial como indicador a ser utilizado na compreensão da problemática da acessibilidade, que foi a hipótese motivadora do trabalho de Souza e Loureiro (2018).

Salienta-se que o parâmetro $\alpha_{n}$ na Equação 1 é a utilidade marginal da renda usada para transformar o logsum em uma medida monetária do valor esperado do excedente do consumidor. No caso de uma função de utilidade aditiva e linear que inclui a tarifa ou custo da viagem, o correspondente coeficiente é a utilidade marginal da renda $\alpha_{n}$. Esta transformação do logsum em valor monetário evita que a estimativa da acessibilidade ou do excedente do consumidor, a partir da aplicação do modelo de utilidade, seja afetada pelo fator escala do erro que não é estimado. De acordo com Jong et al. (2007), a formulação do logsum depende da premissa de que a utilidade marginal da renda é constante em relação a renda. Como o interesse é na diferença do excedente do consumidor e não no seu valor absoluto, argumenta-se que a formulação da Equação 1 pode ser usada para caracterizar o impacto na acessibilidade devido a políticas ou intervenções em transportes cujo impacto varie pouco com a renda dos indivíduos, como é o caso de 
intervenções nos sistemas de transporte público no Brasil que impacta determinado grupo social de uma mesma classe de renda.

$\mathrm{Na}$ formulação da Equação 1, portanto, a percepção dos indivíduos pode ser incorporada em relação aos atributos do sistema de transporte por meio do logsum. Souza e Loureiro (2018) argumentam que o indicador proposto é adequado para analisar os quatro tipos de problemas de desigualdades na acessibilidade definidos por Garcia et al. (2018). Eles argumentam que a análise espacial deste indicador, a sua relação com outras medidas de desempenho da rede, assim como a análise de caracterização da percepção dos usuários, permitem finalmente caracterizar as diferenças nos níveis de acessibilidade da rede espacialmente, não só para um mesmo grupo, mas para diferentes grupos sociais e modos distintos de transportes. Os autores demonstram a aplicabilidade do conjunto de indicadores proposto para caracterizar desigualdades espaciais (Problema tipo I) nos níveis de acessibilidade dos usuários do sistema de transporte público da RMF. Acredita-se que esses indicadores também sejam adequados para caracterizar os ganhos nos níveis de acessibilidade percebida devido a intervenções no sistema de transporte para um dado grupo social, conforme será descrito na Seção 4, a seguir.

\section{PROPOSTA METODOLÓGICA DE MODELAGEM E CARACTERIZAÇÃo DA ACESSIBILIDADE PERCEBIDA PELOS USUÁRIOS}

O esforço de pesquisa proposto neste artigo se apoia no trabalho de Souza e Loureiro (2018) e vem contribuir no método de caracterização da mudança de acessibilidade urbana devido a intervenções nos sistemas de transportes e uso do solo, com base em indicadores que incorporam a percepção dos indivíduos. Assim, é proposta uma metodologia de caracterização do impacto na acessibilidade percebida que tem quatro etapas principais (Figura 1): (i) modelagem das preferências dos indivíduos; (ii) análise de caracterização da percepção dos indivíduos; (iii) modelagem de indicadores para caracterizar problemas de acessibilidade; e (iv) caracterização dos benefícios na acessibilidade. 0 método é aplicado para avaliar como a mudança na oferta do sistema de transportes pode impactar nos níveis de acessibilidade experimentados pela população usuária do sistema de transporte público, tendo como estudo de caso a análise do impacto na acessibilidade causado pelo início da operação das linhas Sul e Oeste do Metrofor na RMF.

0 método proposto de caracterização dos benefícios na acessibilidade percebida é fundamentado não apenas na modelagem dos indicadores, mas também na análise da percepção dos indivíduos sobre a oferta do sistema de transportes, ou seja, suas percepções sobre as ineficiências da rede de transportes (Garcia et al., 2018), assim como sobre a distribuição das atividades no meio urbano. Portanto, este estudo apresenta contribuição tanto no método de caracterização da acessibilidade, como na compreensão do fenômeno da acessibilidade no meio urbano, por meio do conhecimento da percepção de diferentes grupos sociais de usuários, obtido a partir da modelagem comportamental baseada em modelos de escolha discreta. 


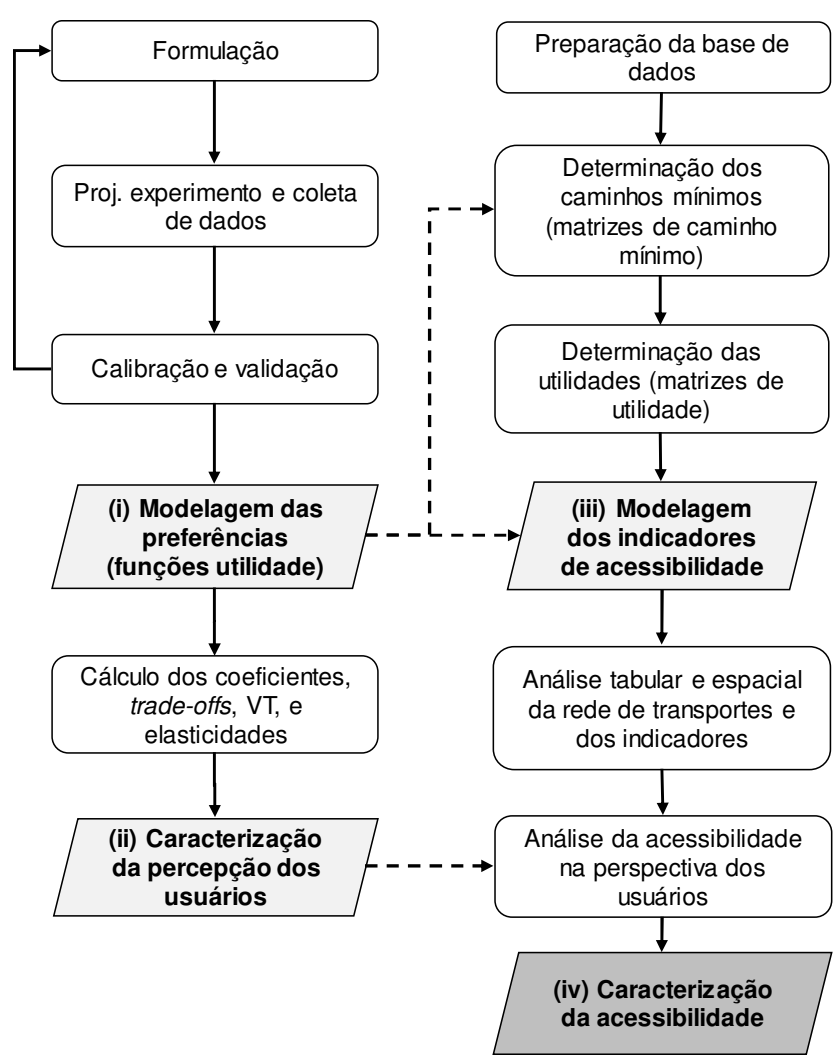

Figura 1. Método proposto de caracterização dos ganhos na acessibilidade percebida

\subsection{Modelagem das características individuais ou de grupos sociais}

As percepções dos indivíduos com relação ao sistema de transportes e a distribuição das atividades no meio urbano podem ser capturadas a partir de modelos de escolha discreta. Neste trabalho, considera-se que a distribuição das atividades é conhecida, tendo como ênfase a modelagem da percepção dos indivíduos com relação ao acesso às atividades, que corresponde ao quanto os usuários são sensíveis às diferentes parcelas dos tempos de deslocamento na rede de transportes.

\subsubsection{Modelo (funções utilidade)}

Em geral, para avaliar a percepção dos indivíduos sobre os diferentes modos numa rede de transportes, definem-se modelos de utilidade como uma função linear nos parâmetros entre a utilidade e os atributos das alternativas, conforme Equação 2.

$$
V_{n i}=\alpha_{i}+\beta_{1} \cdot X_{1}+\beta_{2} \cdot X_{2}+\beta_{3} \cdot X_{3}+\beta_{4} \cdot X_{4}+\beta_{5} \cdot X_{5}
$$

Em que $V_{n i}$ é a utilidade sistemática, $\alpha_{i}$ é a constante específica do modo $i$, as variáveis $X_{j}$, para cada atributo $j$, representam a impedância ao deslocamento e são, respectivamente, o custo $\left(X_{1}\right)$, tempo de caminhada $\left(X_{2}\right)$, tempo de espera $\left(X_{3}\right)$, número de transbordos $\left(X_{4}\right)$ e tempo de viagem $\left(X_{5}\right)$. Os parâmetros, $\beta_{j}$, representam a sensibilidade dos indivíduos a cada atributo $j$ da viagem.

A formulação da Equação 2 é, portanto, uma formulação genérica que incorpora a percepção dos indivíduos sobre as diferentes parcelas do deslocamento. Essas funções utilidade podem ser estimadas a partir da definição de modelos do tipo logit, que são calibrados e validados com base em dados de Preferência Revelada (PR) ou Declarada (PD). Tem-se como ênfase neste trabalho o uso e a análise destes modelos comportamentais para caracterizar a acessibilidade dos 
indivíduos na rede. Para um aprofundamento sobre técnicas de projeto de experimento, coleta de dados, calibração e validação de modelos de escolha discreta do tipo logit, recomendam-se os trabalhos de Louviere et al. (2010) e Train (2009).

A partir da análise das funções utilidade e dos indicadores gerados será possível compreender como os usuários percebem a oferta do sistema de transportes e como isto pode influenciar na sua percepção da acessibilidade. Vale ressaltar que parâmetros específicos sobre os atributos de tempo e custo das alternativas podem ser definidos para representar diferenças de percepção dos indivíduos conforme a alternativa.

\subsubsection{Caracterização da percepção dos usuários}

Esta etapa consiste na análise dos resultados obtidos com a modelagem da escolha discreta. Os próprios coeficientes das funções utilidade representam o grau de importância de cada atributo no processo de tomada de decisão. Os valores destes parâmetros indicam o quanto os grupos de indivíduos são sensíveis a determinada variação nos atributos de tempo ou custo da viagem. Quanto maior o valor destes parâmetros, maior será a sensibilidade dos indivíduos a qualquer variação dos atributos correspondentes, com efeito direto na escolha da alternativa de transportes.

As preferências dos indivíduos em relação aos atributos das alternativas podem ser melhor analisadas a partir de medidas de compensação ou trade-off entre os atributos, que correspondem ao quanto um indivíduo estaria disposto a comprometer a sua utilidade em relação a um dado atributo em compensação pela melhora em outro atributo, mantendo-se constante a utilidade. No caso de uma função utilidade linear, o trade-off entre os atributos $x_{1}$ e $x_{2}$, dado pela Equação 3, corresponde ao quanto se está disposto a perder de $x_{2}$ para ganhar uma unidade de $x_{1}$. Se estes atributos forem tempo e custo, respectivamente, tem-se o valor percebido do tempo, ou $V T=\beta_{\text {tempo }} / \beta_{\text {custo }}$.

$$
\text { trade_off }=\frac{\beta_{1}}{\beta_{2}}
$$

A análise dos coeficientes e das medidas de compensação permite identificar quais os atributos mais importantes segundo a percepção dos indivíduos que utilizam o sistema. Os métodos de estimação dos parâmetros dos modelos de utilidade aleatória não permitem a comparação entre diferentes grupos ou diferentes funções de utilidade. Já as estimativas dos trade-offs, além de representarem a taxa marginal de substituição entre atributos, também permitem comparações entre modelos diferentes ou grupos de indivíduos diferentes.

Por fim, deve-se estimar a elasticidade dos atributos mais importantes segundo a percepção dos indivíduos, trata-se das elasticidades direta e cruzada da probabilidade de escolha. Como os modelos de escolha discreta do tipo logit são modelos não-lineares, para avaliar o efeito da variação dos atributos sobre as probabilidades de escolhas dos indivíduos é necessário determinar medidas de elasticidade. A Equação 4 representa a elasticidade marginal do individuo $n$ da probabilidade de escolher a alternativa $i$ em relação à variação do atributo $x$; enquanto a Equação 5 representa a elasticidade cruzada e a Equação 6 representa a elasticidade agregada que é a elasticidade individual ponderada pela probabilidade de escolha, $P_{n}(i)$, ou seja, agregada pelo método de enumeração amostral. Quanto maior a elasticidade, maior o efeito da variação de um determinado atributo sobre as probabilidades de escolha.

$$
\begin{gathered}
E_{i x_{n i}}=\beta_{x} x_{n i}\left(1-P_{n i}\right) \\
E_{i x_{n j}}=\beta_{x} x_{n j} P_{n j}
\end{gathered}
$$




$$
E_{w}=\frac{\sum_{n=1}^{N} P_{n}(i) E_{i x_{n i}}}{\sum_{n=1}^{N} P_{n}(i)}
$$

\subsection{Determinação da acessibilidade}

A partir dos modelos de utilidade das preferências dos indivíduos sobre as opções de transportes, determina-se a percepção agregada (excedente do consumidor calculada pelo indicador logsum) em diferentes unidades espaciais (por exemplo, zonas de tráfego). A mesma formulação do indicador proposta por Souza e Loureiro (2018) pode ser utilizada para medir as mudanças na acessibilidade devido às intervenções no sistema de transportes, assumindo como medida de atratividade às zonas de destino a própria distribuição espacial das atividades. Portanto, a acessibilidade foi medida por zona de tráfego de origem das viagens, conforme a Equação 1.

\subsubsection{Determinação dos caminhos mínimos e das matrizes de utilidade}

Para o cálculo do indicador de acessibilidade é necessária a aplicação dos modelos de utilidade aleatória para cada zona de origem das viagens. Considerando que em cada zona de origem é possível a realização de viagens para diferentes zonas de destino, e por meio de diferentes modos ou rotas, faz-se necessário primeiro determinar as diferentes opções de transportes juntamente com seus atributos (tempos e custos de deslocamento) para cada par origem-destino (OD). Dada a impossibilidade de enumerar todas as opções de transportes, optou-se por um método de geração de caminhos mínimos entre os pares OD (Souza e Loureiro, 2018), tendo como impedância ao deslocamento a utilidade de cada rota dada pelo modelo da Equação 2. Diferentes caminhos possíveis e multimodais devem ser obtidos para cada par OD, variando o VT de cada parcela do deslocamento e o número de transbordos realizados por rota, que servirão para o cálculo do indicador de acessibilidade.

\subsubsection{Indicador de acessibilidade espacial}

Para determinar a contribuição da nova alternativa modal se opta pela abordagem utilizada no trabalho de Handy e Niemeier (1997), no qual se definem dois cenários: um com todos os modos de transportes e outro com todos os modos exceto aquele que se quer estimar a contribuição na acessibilidade. Determinou-se assim a acessibilidade das zonas de origem para cada cenário, com (Cenário 1) e sem o metrô (Cenário 0), conforme a Equação 1. Assim, o impacto na acessibilidade para um dado grupo de indivíduos $n$ de um dado par OD, ou variação do excedente do consumidor, $E C_{n}$, devido ao metrô é medido conforme a Equação 7:

$$
\Delta E\left(E C_{n}\right)=\left(\frac{1}{\alpha_{n}}\right)\left[\ln \left(\sum_{i} e^{V_{i n}^{1}}\right)-\ln \left(\sum_{i} e^{V_{i n}^{0}}\right)\right]
$$

\subsection{Análise da acessibilidade na perspectiva dos usuários}

Esta etapa trata de consolidar as informações geradas nas etapas anteriores em uma análise de caracterização da acessibilidade na perspectiva dos usuários, que será a base posteriormente para o diagnóstico, encerrando a compreensão sobre o impacto na acessibilidade devido à intervenção urbana. Como discutido, optou-se por trabalhar com um indicador espacial da acessibilidade percebida pelos usuários, conforme Equação 1. Este indicador permite caracterizar espacialmente a acessibilidade dos usuários do sistema de transporte urbano. Assim, pretendese avaliar a variação espacial da acessibilidade com e sem a nova alternativa modal, relacionando a variação observada com as características das preferências dos indivíduos que utilizam 
o sistema (conforme análise da função utilidade da Equação 2) e as características físicas e operacionais da rede de transportes; permitindo assim identificar a contribuição de determinado modo. Esta análise será realizada a partir da elaboração de mapas temáticos e da análise de inferência de indicadores locais de dependência espacial (LISA maps). A análise de dependência espacial tem como intuito identificar aglomerados de zonas bem servidas (com elevados níveis de acessibilidade) e zonas mal servidas (com baixos níveis de acessibilidade), com e sem a alternativa a ser implantada. Esta última análise permite caracterizar a influência do novo modo em diferentes zonas de tráfego, de forma a identificar zonas onde ocorreram ganhos significativos na acessibilidade com a inserção deste modo, assim como zonas onde estes ganhos não foram significativos, ou ainda zonas onde ocorreu redução no nível de acessibilidade.

\section{CARACTERIZAÇÃO DA ACESSIBILIDADE PROPORCIONADA PELO METRÔ}

Para caracterizar a contribuição do sistema metroferroviário, considerando o seu estágio de operação em agosto de 2018, utilizou-se os dados disponíveis sobre a RMF e seus sistemas de transportes. 0 sistema metropolitano de transportes da RMF contempla 13 municípios que são atendidos por cerca de 400 rotas de ônibus / vans, com um pouco mais de 2.400 veículos distribuídos entre o serviço urbano de Fortaleza, os serviços urbanos de Caucaia, Maracanaú e Maranguape, e o serviço metropolitano (entre municípios da RMF), bem como pelas linhas sul e oeste do Metrofor (Figura 2). 0 sistema como um todo atende cerca de 1.200.000 deslocamentos por dia útil. Destes deslocamentos, o sistema urbano de Fortaleza é responsável por cerca de $80 \%$. As tarifas praticadas seguem regras diferentes entre os sistemas. Para o sistema urbano de Fortaleza, tem-se tarifa única, enquanto para os outros sistemas urbanos e para o metrô a tarifa é definida por linha. Já no sistema metropolitano, a tarifa é definida por anéis tarifários.

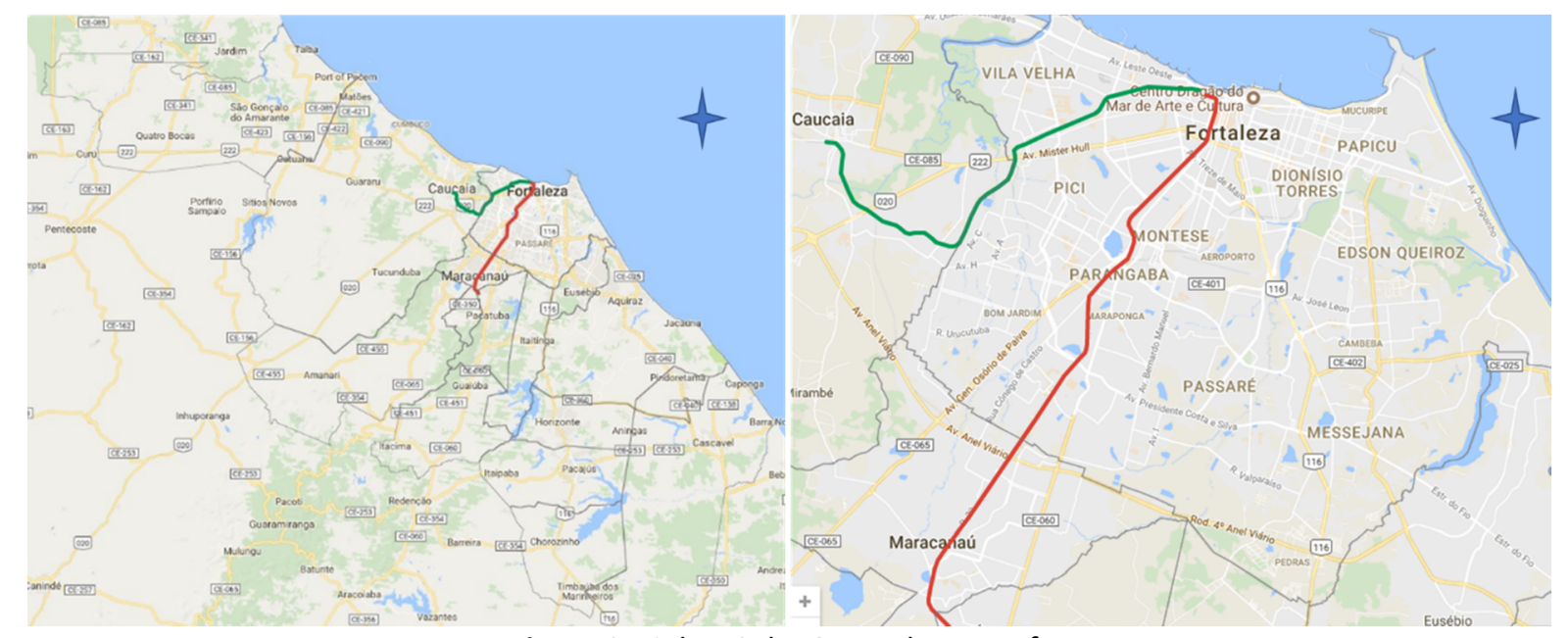

Figura 2. Linhas Sul e Oeste do Metrofor

\subsection{Descrição das bases de dados utilizadas}

Foram utilizadas duas bases de dados: a primeira para se estimar as funções utilidade; e a segunda para modelar a oferta de transporte público na RMF. A primeira tem origem em estudo contratado pelo Metrofor (ASTEF, 2006) com o objetivo de identificar o processo de escolha dos seus potenciais usuários. Na oportunidade, foram realizadas pesquisas que identificaram o perfil destes usuários e seus deslocamentos, assim como pesquisas de preferência declarada. Por 
sua vez, a segunda base contempla dados georreferenciados da RMF, tendo sido utilizada em estudo desenvolvido pela agência reguladora estadual (ARCE e Concremat, 2013) e atualizada para representar as atuais impedâncias dos deslocamentos.

No projeto de experimento da pesquisa de PD, definiu-se como conjunto de escolha quatro modos: metrô, ônibus urbano, ônibus metropolitano e trem. Ao total foram consideradas seis variáveis de transporte como influentes no processo de escolha: custo da viagem; tempo de viagem; tempo de espera; distância de caminhada; condições de caminhada e número de transbordos, cada uma delas com dois níveis. Para reduzir o número cenários, aplicou-se um projeto fatorial fracionado, em que para cada entrevistado eram apresentados oito cartões, cada um com duas alternativas e dois atributos cada. Ao total foram entrevistados 1.638 usuários que geraram 9.440 observações válidas.

\subsection{Modelagem das características individuais ou de grupos sociais}

Seguindo o método proposto, foram especificados e calibrados modelos para determinação das funções utilidade. Vale ressaltar que, diferente do trabalho de Souza e Loureiro (2018) que especificaram um modelo de utilidade com parâmetros genéricos para demonstrar a utilização dos indicadores propostos, neste trabalho a análise da percepção dos usuários é uma etapa essencial para caracterização da acessibilidade. Portanto, novos modelos foram formulados conforme o contexto de análise.

\subsubsection{Modelo (funções utilidade)}

Na formulação do modelo, após testar algumas especificações, considerou-se as mesmas alternativas definidas no projeto de experimento: metrô, ônibus metropolitano, ônibus urbano e trem. Para os atributos, foram considerados aqueles representativos da impedância dos deslocamentos realizados em transporte público, ou seja, custo da viagem, tempo de viagem, tempo de espera, tempo de caminhada e número de transbordos. Com relação ao tipo de função, assumiu-se uma função de utilidade linear nos parâmetros, conforme Equação 8, adotando-se como modelo de escolha um modelo logit multinomial.

$$
U_{i}=\alpha_{i}+\beta_{c} x_{c, i}+\beta_{\text {cam }} x_{c a m, i}+\beta_{\text {tran }} x_{\text {tran }, i}+\beta_{\text {espe }, i} x_{\text {espe }, i}+\beta_{\text {viag }, i} x_{\text {viag }, i}+\varepsilon_{i}
$$

Em que: $i=$ modo de transporte, $\alpha_{i}=$ constante específica do modo $i, c=$ custo do deslocamento (tarifa), $c a m$ = tempo de caminhada, $\operatorname{tran}=$ quantidade de transbordos, espe = tempo de espera, viag = tempo de viagem, = componente aleatória. Na especificação da Equação 8, assumiu-se que a percepção dos usuários sobre o tempo de espera e o tempo de viagem é específica para os modos rodoviários (ônibus urbano e metropolitano) e para os modos ferroviários (metrô e trem). Assim, assumiu-se parâmetros específicos, $\beta_{\text {espe, } i}$ e $\beta_{\text {viag,i }}$, por modo ferroviário e rodoviário, do tempo de espera e do tempo de viagem. Considerando o contexto de análise do impacto do metrô na acessibilidade, esta premissa, portanto, considera as diferenças de percepção entre os usuários de transporte metroferroviário e o transporte rodoviário.

A Tabela 1 apresenta os resultados da calibração dos parâmetros do modelo especificado na Equação 8. Os sinais das constantes específicas indicam uma maior preferência pelo modo metrô. Verifica-se também que todos os coeficientes apresentaram sinal negativo, como esperado. Além disso, o elevado valor da razão de verossimilhança de 564,14, comparando com o valor de qui-quadrado crítico de 16,8, ao nível de 1\% de significância, indica haver evidências de que os atributos considerados explicam as preferências dos indivíduos quanto ao modo de transporte coletivo. 
Para analisar a hipótese de que os parâmetros de tempo de espera e de viagem devem ser específicos por modo, o modelo proposto com parâmetros específicos foi comparado a um modelo com todos os parâmetros genéricos. 0 teste da razão de verossimilhança resultou numa estatística teste de 36,0, que comparado a um valor de qui-quadrado crítico de 11,3, ao nível de 1\% de significância, indica haver evidências de que existe diferença de percepção conforme o modo de transporte. Além disso, os indicadores AIC (Akaike information criterion), de 12573 e 12542, e BIC (Bayesian information criterion), de 12622 e 12614, para o modelo com parâmetros genéricos e para o modelo com parâmetros específicos por modo, respectivamente, também apontam que o modelo com parâmetros específicos (com valores mais baixos de AIC e BIC) é mais adequado.

É importante ressaltar que o valor baixo do $\mathrm{R}^{2}$ pode indicar que outros atributos que não foram considerados podem ter influência na decisão de escolha do modo. Pode indicar também alguma heterogeneidade entre os indivíduos que também não foi considerada. Como o principal objetivo do artigo é caracterizar a contribuição da implantação do metrô na acessibilidade dos indivíduos por meio de uma medida de acessibilidade que considere aspectos comportamentais, optou-se, neste momento, por especificar um modelo viável de ser estimado com dados disponíveis e que pudesse ser usado para caracterizar o ganho, de forma espacial, na acessibilidade com a implantação do metrô.

Tabela 1 - Resultados da calibração

\begin{tabular}{lrrr}
\hline Coeficiente & Estimativa & Erro padrão & Estatística $\boldsymbol{t}$ \\
\hline$\alpha_{i}$ & & & \\
Metrô & 0 & Referência & \\
Ônibus metropolitano & $-0,0136$ & 0,0417 & $-0,33$ \\
Ônibus urbano & $-0,0689$ & 0,0389 & $-1,77$ \\
Trem & $-0,355^{*}$ & 0,0359 & $-9,90$ \\
\hline$\beta_{c}$ & $-0,681^{*}$ & 0,0362 & $-18,82$ \\
\hline$\beta_{\text {cami }}$ & $-0,165^{*}$ & 0,0218 & $-7,58$ \\
\hline$\beta_{\text {jespe }}$ & & & \\
Ferroviário & $-0,0194^{*}$ & 0,00767 & $-2,53$ \\
Rodoviário & $-0,0366^{*}$ & 0,0116 & $-3,17$ \\
\hline$\beta_{\text {tran }}$ & $-0,482^{*}$ & 0,0536 & $-8,99$ \\
\hline$\beta_{\text {jviag }}$ & & & \\
Ferroviário & $-0,017^{*}$ & 0,0027 & $-6,32$ \\
Rodoviário & $-0,0215^{*}$ & 0,00249 & $-8,64$ \\
\hline Logaritmo da verossimilhança: & $-6261,241$ & & \\
Razão de verossimilhança: & 564,136 & & \\
Pseudo R ${ }^{2}$ & 0,043 & & \\
$\mathrm{R}^{2}$ ajustado: & 0,042 & & \\
\hline * significativo ao nível de 1\% & & & \\
\hline
\end{tabular}

\subsubsection{Análise dos coeficientes, trade-offs, VT e elasticidades}

Como visto na Tabela 1, a constante específica do modo trem apresentou valor negativo e significativo; já para os outros modos, a estimativa da constante específica não foi significativa. Esperava-se, entretanto, uma maior preferência ao modo metrô em relação aos demais modos. Uma possível interpretação deste resultado é que o usuário (que a época não conhecia a operação de um metrô, apenas de trem) não diferenciava, a priori, estes modos de transportes (ônibus e metrô). Por outro lado, este mesmo usuário já conhecia a operação do trem, que na época apresentava operação deficitária e, portanto, o considerava como um serviço inferior. Observase também que, em relação às parcelas do tempo de deslocamento, os usuários são em geral 
mais sensíveis ao tempo de caminhada, seguido pelo tempo de espera no modo rodoviário e tempo de viagem no modo rodoviário. Há, portanto, indícios de que os usuários percebam como mais penoso o tempo de espera e no veículo para o modo rodoviário, se comparado ao modo ferroviário, com a caminhada tendo maior penalidade.

Considerando que a variação dos atributos, principalmente do tempo de caminhada e da tarifa, afeta a escolha do modo metrô, determinou-se a elasticidade agregada direta e cruzada da demanda em relação a estes dois atributos. É importante destacar que as elasticidades diretas e cruzadas devem ser interpretadas de forma comparativa entre os atributos, pois os parâmetros do modelo de escolha discreta são estimados juntamente com parâmetro de escala do erro da função de utilidade aleatória. Com relação à tarifa, obteve-se um valor de 0,62 de elasticidade direta da probabilidade de escolha do metrô, e de 0,98; 0,57 e 0,36 de elasticidade cruzada da probabilidade de escolha do metrô com relação a variações da tarifa do ônibus metropolitano, ônibus urbano e trem. Já com relação ao tempo de caminhada obteve-se um valor de 0,04 de elasticidade direta da probabilidade de escolha do metrô, e de 0,05; 0,04 e 0,03 de elasticidade cruzada com relação a variações no tempo de caminhada do ônibus metropolitano, ônibus urbano e trem. Estes resultados mostram que a probabilidade de escolha do modo metrô é relativamente mais influenciada pela tarifa e tempo de caminhada do modo ônibus metropolitano do que sua própria tarifa ou tempo de caminhada.

Com relação aos trade-offs entre os atributos, conforme mostrado na Tabela 2, destaca-se mais uma vez a caminhada como uma etapa do deslocamento considerada pelos usuários como sendo mais penosa do que o período dentro dos veículos. A Tabela 2 mostra que os usuários estão dispostos a aumentar em mais de 9 e 7 minutos o seu tempo de viagem, respectivamente nos modos ferroviário e rodoviário, para reduzir um minuto no tempo de caminhada. Os resultados da Tabela 2 mostram ainda que os usuários estão dispostos em aumentar mais de 20 minutos seu tempo de viagem para reduzir um único transbordo.

Tabela 2 - Trade-off entre variáveis e valor do tempo

\begin{tabular}{|c|c|c|c|c|}
\hline & Trade-off & Trade-off & & \\
\hline Atributos & $\begin{array}{r}\text { com o tempo de viagem } \\
\text { no ferroviário }\end{array}$ & $\begin{array}{r}\text { com o tempo de viagem no } \\
\text { rodoviário }\end{array}$ & VT percebido & VT percebido** \\
\hline Tempo de caminhada & 9,71 & 7,67 & $R \$ 14,54$ & $R \$ 31,62$ \\
\hline Tempo de espera modo ferroviário & 1,14 & & $\mathrm{R} \$ 1,71$ & $R \$ 3,72$ \\
\hline Tempo de espera modo rodoviário & & 1,70 & $\mathrm{R} \$ 3,22$ & $\mathrm{R} \$ 7,00$ \\
\hline Número de transbordos & $28,35^{*}$ & 22,42 & $\mathrm{R} \$ 0,71$ & $\mathrm{R} \$ 1,54$ \\
\hline Tempo de viagem modo ferroviário & & 0,79 & $\mathrm{R} \$ 1,50$ & $R \$ 3,26$ \\
\hline Tempo de viagem modo rodoviário & 1,26 & & $\mathrm{R} \$ 1,89$ & $\mathrm{R} \$ 4,11$ \\
\hline
\end{tabular}

Já com relação aos resultados do valor do tempo apresentados na Tabela 2, verifica-se mais uma vez o quanto os usuários são sensíveis ao tempo de caminhada, apresentando o maior VT de aproximadamente $\mathrm{R} \$ 31,00 / \mathrm{h}$. Destaca-se também os VTs do tempo de espera e do tempo de viagem no modo rodoviário em relação ao modo ferroviário. 0 valor obtido do tempo de viagem nos modos rodoviários parece coerente se comparado aos resultados levantados por Santos (2012) que encontrou valores (corrigidos para junho de 2019) entre $R \$ 5,57 / h$ e $R \$ 13,94 / h$ para o valor do tempo de usuários de ônibus em algumas cidades brasileiras. A estimativa do VT para os modos rodoviários também condiz com o obtido por Cavalcante (2002), 
que estimou em $R \$ 1,78 / h$ (atualizado em $R \$ 6,01 / h$, para junho de 2019) o valor do tempo de viagem de usuários do transporte coletivo da RMF.

\subsection{Determinação e caracterização espacial da acessibilidade}

Detalha-se a seguir a determinação da acessibilidade por zona de origem, bem como a análise de caracterização espacial destes resultados. Destaca-se que o grupo de interesse neste estudo é de usuários do transporte público, que no Brasil em sua maioria são usuários que se encontram numa mesma classe de renda. Portanto, a medida de acessibilidade proposta na Equação 1 foi aplicada para caracterizar problemas de acessibilidade para um mesmo grupo social. A análise da variação de acessibilidade entre diferentes grupos sociais não é considerada neste artigo.

\subsubsection{Determinação dos caminhos mínimos, matrizes de utilidade e cálculo da acessibilidade}

Inicialmente, foram geradas matrizes de custo, tempo de espera, tempo de caminhada, número de transbordo e tempo no veículo (por modo: transporte urbano de Fortaleza, transporte urbano de outros municípios, transporte metropolitano e linhas sul e oeste do Metrofor), ou seja, um total de nove matrizes. A ferramenta utilizada gerava apenas um caminho mínimo entre zonas e, para fins de acessibilidade via logsum, deve-se considerar múltiplos caminhos que sejam concorrentes. Para gerar esses caminhos, alterou-se alguns parâmetros do método de caminho mínimo. Estes parâmetros foram o número máximo de transbordos permitidos (variando de um a três) e VT (variando em mais e menos 25\% do valor estimado a partir das funções de utilidade calibradas). Assim, teve-se três valores para o número máximo de transbordos e três valores para o VT gerando nove cenários. Considerando-se a existência de nove matrizes por cenário, obteve-se um total de 81 matrizes. Destas matrizes, obteve-se os valores das variáveis que compõem a função utilidade; aplicando-se então esses valores nas funções calibradas, obteve-se as utilidades para cada par OD que serviram para estimativa do logsum. Por fim, aplicando-se a Equação 1, tem-se a acessibilidade das zonas de origem a todas as demais zonas. 0 mesmo procedimento foi realizado desconsiderando o metrô, possibilitando comparar a acessibilidade com e sem metrô, ou seja, a contribuição do mesmo na rede.

\subsubsection{Caracterização espacial da acessibilidade na perspectiva dos usuários}

A Figura 3 apresenta mapas temáticos da diferença de acessibilidade com e sem o Metrofor, ou seja, seu grau de contribuição na melhoria da acessibilidade. Verifica-se que a inserção das linhas sul e oeste aumenta a acessibilidade de quase toda a região, destacando-se Fortaleza onde todas as zonas sofrem influência positiva do metrô. A maioria das zonas que recebem maior contribuição estão em Fortaleza, destacando-se suas regiões sul e oeste. Também se destacam regiões nos municípios de Itaitinga, sul de Maranguape e parte de Pacajus, que são zonas (classificadas como High-Low no LISA map) com aumento significativo de acessibilidade em comparação com as zonas vizinhas. Nota-se também, a partir do LISA map, que as zonas que mais se beneficiam são zonas periféricas ao sul de Fortaleza, ou seja, zonas mais distantes da sua região central. Este resultado evidencia uma melhora nos níveis de acessibilidade da população menos favorecida, ou de baixa renda, que se concentra principalmente nas regiões periféricas da Capital. Estes resultados retratam, portanto, a realidade dos usuários do transporte público que em geral têm que enfrentar longos tempos de deslocamento e realizar transbordos ao longo das viagens. 
Como discutido na etapa de modelagem da percepção dos indivíduos, os transbordos e os tempos de espera e de viagem no sistema rodoviário se constituem como as principais impedâncias aos deslocamentos. Atualmente o sistema rodoviário de transporte urbano de Fortaleza opera com bilhete único, permitindo integração temporal no sistema. Contudo, a rede de transporte público ainda é predominantemente tronco-alimentada, fazendo com que muitas das viagens que se originam nas zonas periféricas da cidade ainda passem pelos terminais físicos de integração, resultando em maiores tempos de viagem e de espera nesses terminais.

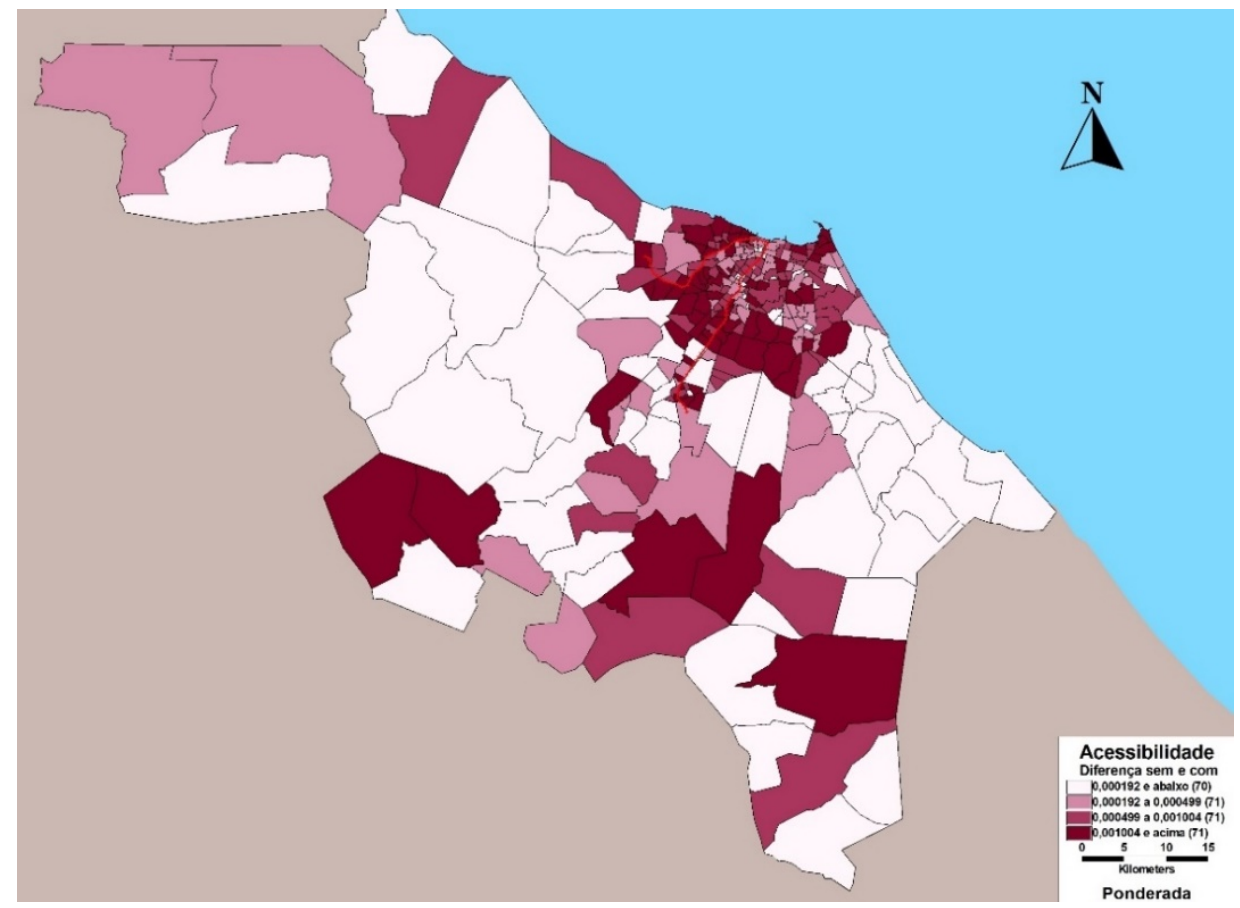

Figura 3. Diferença de acessibilidade sem e com Metrô

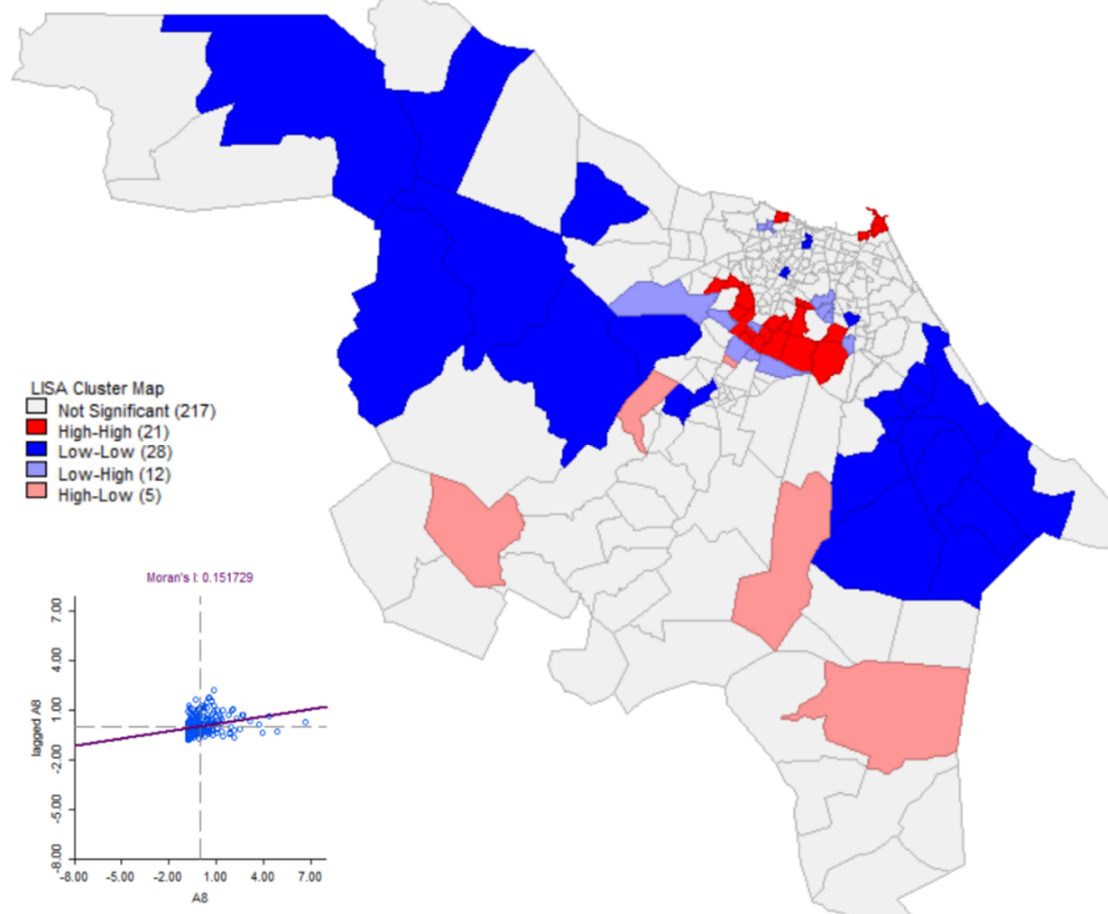

Figura 4. LISA MAP de acessibilidade proporcionada pelo Metrô - diferença de acessibilidade 


\subsection{Análise do impacto na acessibilidade na perspectiva dos usuários}

De forma geral, a acessibilidade piora à medida que se afasta da região central de Fortaleza e da linha sul do metrô. Isto pode estar associado à concentração dos empregos na área central e a uma forte concentração da população nas proximidades da linha sul do metrô. Pode-se observar a contribuição do metrô na maioria das zonas, mesmo naquelas mais distantes da rede metroviária. Este fato pode ser explicado pelas próprias características do indicador (logsum) que valoriza a existência das alternativas mesmo que estas não sejam escolhidas; assim como pelo metrô atender à área com maior atratividade, sendo, portanto, uma boa alternativa de acesso ao centro de Fortaleza.

A percepção dos indivíduos e as características da rede de transportes podem ajudar a caracterizar os ganhos de acessibilidade com o metrô. De maneira geral, as pessoas preferem perder tempo nos deslocamentos dentro dos veículos a caminhar, a esperar ou a realizar transbordos. Isto implica que uma boa distribuição das linhas e pontos de parada / estações, mesmo numa rede com elevados tempos de viagem, pode ser preferida pelos usuários a uma rede com tempo de viagem otimizado mas com má cobertura espacial. Um aspecto a destacar é a diferença de percepção dos atributos de tempo de viagem e espera entre os modos ferroviário e rodoviário. Isto pode explicar o porquê de, nas áreas próximas à região central e afastadas da linha sul do metrô, não se observarem ganhos significativos de acessibilidade. Nestas áreas o efeito da realização de transbordos e do tempo de espera é mais evidente. Já nas áreas mais periféricas, onde a realização de transbordos e tempos de espera em terminais de integração são mais frequentes, percebe-se um maior benefício com a inserção do metrô, já que existe uma preferência dos indivíduos pelo modo ferroviário quando ocorrem transbordos, tempos de espera e períodos longos dentro do veículo. 0 custo da viagem é também atributo considerado importante, em especial para os usuários do transporte ferroviário. Assim, a política de tarifa única aplicada em Fortaleza e a integração subsidiada entre o sistema metropolitano e urbano de Fortaleza favorecem a resultados relativamente bons da acessibilidade em áreas mais distantes.

\section{CONSIDERAÇÕES FINAIS}

No presente trabalho, apresentou-se um método de caracterização da acessibilidade que incorpora a percepção dos usuários sobre o desempenho da rede de transportes e a distribuição das atividades no meio urbano, a partir do conceito da acessibilidade percebida pelos indivíduos. 0 método tem como base uma medida de acessibilidade (logsum) que incorpora tal percepção dos usuários do sistema urbano de transportes, sendo fundamentado em modelos comportamentais de escolha discreta. 0 trabalho, portanto, apresenta contribuição metodológica com relação ao uso de modelagem comportamental nas etapas de caracterização da acessibilidade percebida pelos usuários, como parte da nova abordagem do planejamento de transportes que tem como ênfase a análise da equidade na acessibilidade. Além disso, a aplicação do método no sistema de transportes metropolitano de Fortaleza permitiu caracterizar espacialmente os ganhos de acessibilidade com as linhas Sul e Oeste do Metrofor. Vale destacar que neste estudo foi considerada apenas a percepção dos indivíduos sobre o sistema de transportes, sendo o sistema de atividades considerado como exógeno.

A análise de caracterização espacial em área da acessibilidade promovida pela implantação do Metrofor na RMF mostrou que há contribuição do metrô na maioria das zonas de tráfego, mesmo naquelas mais distantes das linhas metroviárias. Em particular, foi possível identificar 
zonas onde ocorreram ganhos significativos na acessibilidade com a inserção das linhas de sul e oeste; como também zonas onde estes ganhos não foram significativos. A análise da percepção dos indivíduos sobre a oferta dos sistemas de transportes, com base nos modelos comportamentais ou de utilidade aleatória propostos, permitiu caracterizar esses ganhos de acessibilidade. De um modo geral, argumenta-se que a variação espacial da acessibilidade percebida pelos usuários do transporte público na RMF pode ser melhor explicada pela percepção dos indivíduos sobre os atributos de tempo de caminhada, tempo de espera e transbordos dos deslocamentos. Apontou-se também que a diferença de percepção dos indivíduos entre os modos rodoviário e metroviário, em relação aos atributos tempo de espera e de viagem, explicaria os ganhos significativos de acessibilidade gerados, principalmente em zonas periféricas ou mais afastadas da região central de Fortaleza, onde estão mais concentrados os empregos. Estas, entre outras características dos usuários, permitem compreender a contribuição do Metrofor na acessibilidade, em especial nas áreas periféricas.

Por fim, vale ressaltar que pode haver diferença de percepção dos indivíduos, principalmente de classes sociais diferentes, com relação aos atributos de escolha do modo e a outros atributos não observados, como conforto e segurança, que pode influenciar na variação da probabilidade de escolha. Além disso, outras estruturas (e.g., Nested Logit) de modelo podem ser especificadas caso existam evidências de que na percepção dos usuários os atributos não observados sejam similares entre alternativas. Esta percepção sobre os atributos considerados e a similaridade nas alterativas quanto a outros aspectos não considerados podem também variar espacialmente, conforme local de origem da viagem. Portanto, outras especificações de modelos de escolha discreta e indicadores de acessibilidade do tipo logsum que considerem a heterogeneidade entre indivíduos e a similaridade entre alternativas constitui-se como importante direção para trabalhos futuros.

\section{REFERÊNCIAS}

ARCE e Concremat (2013) Atualização da base de dados georreferenciados do STIP-CE. Agência Reguladora dos Serviços Publicos do Estado do Ceará. Fortaleza, CE.

ASTEF (2006) Pesquisa de identificação dos usuários da alternativa de implantação dos trechos Vila das Flores / Couto Fernandes e Couto Fernandes / João Felipe - Linha Sul do Metrofor. Fortaleza, CE.

Banister, D. (2008) The sustainable mobility paradigm. Transport Policy, 15, 73-80. DOI: 10.1016/j.tranpol.2007.10.005.

Bhat, C., Handy, S., Kockelman, K., Mahmassani, H., Chen, Q., e Weston, L. (2000) Urban Accessibility Index: Literature Review. Texas Department of Transportation, University of Texas, Austin.

Börjesson, M., Jonsson, R.D., Berglund, S., e Almström, P. (2014) Land-use impacts in transport appraisal. Res. Transp. Econ. DOI: 10.1016/j.retrec.2014.09.021.

Cascetta, E. (2009) Transportation systems analysis: models and applications. (2a.). Springer, New York.

Cascetta, E., Cartenì, A., e Montanino, M. (2013). A new measure of accessibility based on perceived opportunities. Procedia Social and Behavioral Sciences, 87, 117-132. DOI: 10.1016/j.sbspro.2013.10.598

Cavalcante, R. A. (2002) Estimativa das penalidades associadas com os transbordos em sistemas de integrados de transporte público. Dissertação. Universidade Federal do Rio de Janeiro, COPPE.

Garcia, C. S. H. F., Macário, M. R. M. R., Menezes, E. D. A. G., e Loureiro, C. F. G. (2018) Strategic Assessment of Lisbon's Accessibility and Mobility Problems from an Equity Perspective. Networks and Spatial Economics, 1-25. DOI: 10.1007/s11067018-9391-4.

Geurs, K. T., De Montis, A., e Reggiani, A. (2014) Recent advances and applications in acessibility modelling. Computers, Environmenjt and Urban Systems, n. 49, p. 82-85.

Geurs, K.T., e van Wee, B., (2004) Accessibility evaluation of land-use and transport strategies: review and research directions. J. Transp. Geogr. 12, 127-140. DOI: 10.1016/j.jtrangeo.2003.10.005.

Geurs, K. T., Zondag, B., de Jong, G., e de Bok, M. (2010) Accessibility appraisal of land-use/transport policy strategies: More than just adding up travel-time savings. Transportation Research Part D-Transport and Environment, 15(7), 382-393. DOI: 10.1016/j.trd.2010.04.006

Handy, S. (2005) Planning for accessibility: in theory and in practice. Access to destinations. 131-147, Elsevier Ltd. 
Handy, S. L., e Niemeier, D. A. (1997) Measuring accessibility: an exploration of issues and alternatives. Environment and Planning A, 29, 1175-1194. DOI: 10.1068/a291175

Hansen, W. G. (1959) How Accessibility Shapes Land Use. Journal of the American Planning Association, v. 25, n. 2, p. 73-76.

Jong, G., Daly, A., Pieters, M., e van der Hoorn, T. (2007) The logsum as an evaluation measure: Review of the literature and new results. Transportation Research Part A: Policy and Practice, 41, 874-889. DOI: 10.1016/j.tra.2006.10.002

Jong, G., Pieters, M., Daly, A., Graafland-Essers, I., Kroes, E., e Koopmans, C. (2005) Using the logsum as an evaluation measure: literature and case study. Santa Monica: Calif.: RAND Corporation.

Koenig, J. G. (1980) Indicators of urban accessibility: Theory and application. Transportation (Amst), v. 9, n. 2, p. $145-172$. DOI: $10.1007 /$ BF00167128.

Lopes, A. S., Loureiro, C. F. G., e Van Wee, B. (2018) LUTI operational models review based on the proposition of an a priori ALUTI conceptual model. Transport Reviews, p. 1-22. DOI:10.1080/01441647.2018.1442890.

Louviere, J. J., Hensher, D. A., e Swait, J. D. (2010) Stated choice mehods: analysis and application. Cambridge University Press.

Miller, E. J. (2018) Accessibility : measurement and application in transportation planning. Journal Transport Reviews, v. 38, n. 5., p. 551-555. DOI: 10.1080/01441647.2018.1492778.

Niemeier, D. A. (1997) Accessibility: An evaluation using consumer welfare. Transportation (Amst), v. 24, n. 4, p. $377-396$. DOI: $10.1023 / A: 1004914803019$.

Santos, C. C. (2012) O Valor do Tempo na Avaliação de Projetos de Transportes. Dissertação. Universidade Federal do Rio Grande do Sul.

Small, K. A., e Rosen, H. S. (1981) Applied welfare economics with discrete choice models. Econometrica, 49(1), 105-130. DOI:10.2307/1911129.

Soares, F. D. P., Loureiro, C. F. G., e Miller, E. J. (2018) Diagnosis: A Problem-Oriented Approach to Urban Transportation Planning. Transportation Research Board 97th Annual Meeting. Washington DC.

Souza, H. H. H. de, e Loureiro, C. F. G. (2018) Proposta de construção de indicador de caracterização da acessibilidade na região metropolitana de Fortaleza. Transportes, p. 129-143. DOI: 10.14295/transportes.v26i4.1546.

Train, K. E. (2009) Discrete Choice Methods with Simulation. 2a ed. Cambridge University Press.

van Wee, B. (2016) Accessible accessibility research challenges. Journal of Transport Geography, v. 51, p. 9-16. DOI:10.1016/j.jtrangeo.2015.10.018. 